Article

\title{
Ensemble CNN to Detect Drowsy Driving with In-Vehicle Sensor Data
}

\author{
Yongsu Jeon ${ }^{1} \mathbb{D}$, Beomjun Kim ${ }^{2} \mathbb{D}$ and Yunju Baek ${ }^{1, *}$ \\ 1 Department of Information Convergence Engineering, Pusan National University, Busan 46241, Korea; \\ ysjeon@pusan.ac.kr \\ 2 Department of Smart Software, Yonam Institute of Technology, Jinju 52821, Korea; bjkim@yc.ac.kr \\ * Correspondence: yunju@pusan.ac.kr
}

Citation: Jeon, Y.; Kim, B.; Baek, Y. Ensemble CNN to Detect Drowsy Driving with In-Vehicle Sensor Data. Sensors 2021, 21, 2372. https:// doi.org/10.3390/s21072372

Academic Editors: Mario

Martínez-Zarzuela and David González Ortega

Received: 9 February 2021

Accepted: 26 March 2021

Published: 29 March 2021

Publisher's Note: MDPI stays neutral with regard to jurisdictional claims in published maps and institutional affiliations.

Copyright: (C) 2021 by the authors. Licensee MDPI, Basel, Switzerland. This article is an open access article distributed under the terms and conditions of the Creative Commons Attribution (CC BY) license (https:// creativecommons.org/licenses/by/ $4.0 /)$.

\begin{abstract}
Drowsy driving is a major threat to the safety of drivers and road traffic. Accurate and reliable drowsy driving detection technology can reduce accidents caused by drowsy driving. In this study, we present a new method to detect drowsy driving with vehicle sensor data obtained from the steering wheel and pedal pressure. From our empirical study, we categorized drowsy driving into long-duration drowsy driving and short-duration drowsy driving. Furthermore, we propose an ensemble network model composed of convolution neural networks that can detect each type of drowsy driving. Each subnetwork is specialized to detect long- or short-duration drowsy driving using a fusion of features, obtained through time series analysis. To efficiently train the proposed network, we propose an imbalanced data-handling method that adjusts the ratio of normal driving data and drowsy driving data in the dataset by partially removing normal driving data. A dataset comprising $198.3 \mathrm{~h}$ of in-vehicle sensor data was acquired through a driving simulation that includes a variety of road environments such as urban environments and highways. The performance of the proposed model was evaluated with a dataset. This study achieved the detection of drowsy driving with an accuracy of up to $94.2 \%$.
\end{abstract}

Keywords: intelligent vehicle; safety system; driver status monitoring; drowsy driving detection; ensemble CNN

\section{Introduction}

In the vehicle industry, technologies for driver safety and convenience such as autonomous vehicles, intelligent vehicles, and advanced driver assistance systems are being actively researched [1-3]. Autonomous vehicles has garnered significant attention from society as well.

SAE International has defined a six-level rating under the J3016 standard to measure the level of autonomous vehicle technology. Currently, various studies are being conducted for partial driving automation systems (level 2) and conditional driving automation systems (level 3) [4,5]. In level 2 or level 3 autonomous driving systems, it is essential to implement the takeover request (TOR) method, wherein the system transfers control authority to the driver. A number of studies [6,7] are being conducted for appropriate TOR methods. One major limitation with the TOR method involves predicting the driver's response time to the request. For this prediction, a driver status monitoring method that monitors drowsy driving or fatigue driving is required.

Drowsy driving is responsible for 130,000 deaths worldwide annually [8]. According to the National Highway Traffic Safety Administration (U.S. Department of Transportation), about 1000 deaths and more than 90,000 injuries each year are caused by drowsy driving [9]. According to the 2020 announcement by the Korea Traffic Accident Analysis System (TAAS), more than 2500 cases of drowsy driving accidents occur every year. Consequently, drowsy driving has been identified as a major cause of traffic accidents that have occurred on highways over the past 10 years [10]. 
Previous studies conducted to detect drowsy driving employed methods that can be divided into three categories based on the data used for detection. Three types of data are commonly used: image data, biosignal data, and sensor data.

Image data-based methods acquire data by installing cameras inside the vehicle. To detect drowsy driving, information is generated by characteristics such as the percentage of eyelid closure (PERCLOS), eye movement, and face direction through an image processing method [11-17]. Since image data directly represents the behavior of the driver during drowsy driving, studies employing image data generally display excellent performance. However, this approach is vulnerable to changes in vehicle illumination and camera lens pollution, and the performance is degraded depending on the driver's attire such as hats and glasses. Since the position and shooting angle of the camera have an important effect on performance, it is necessary to have it installed by a specialist.

Biosignal data-based methods acquire data through wearable devices. Sensors such as electrocardiograms (ECG) [18-20], electromyography (EMG) [21-23], and electroencephalography (EEG) [24-26] are mainly used. Studies that use biosignal data directly represent the body state of the driver, and they also show excellent performance. However, there is a structural limitation in terms of attaching the device to the body and noise is generated when the contact or grounding is poor, which can affect the reliability of the method.

Sensor data-based methods use two types of sensor data: in-vehicle sensor data and external sensor data. The methods can extract statistical $[27,28]$ and time series features $[29,30]$ from the acquired data. They can detect changes in vehicle movement patterns using the extracted features and traditional machine learning techniques such as random forest [31] and support vector machine (SVM) [32]. In general, they have a low detection accuracy and performance changes drastically depending on driving habits or road types. Because they utilize indirect information such as sudden turns and vehicle movement patterns, it is difficult to determine whether such changes occur due to drowsy driving, erratic driving behavior, or even different road types. However, even if the data acquisition method or install location changes, the amount of change in both the in-vehicle sensor and the external sensor is small. In other words, sensor-based systems are relatively easy to install, making it more practical than previous approaches.

For the same reason as mentioned above, the methods that use sensor data require data acquisition from an actual vehicle. However, it is extremely dangerous to acquire sensor data from an actual vehicle during drowsy driving, and as such, previous studies have collected the data via driving simulations. In numerous studies, a limited simulation environment [33] has been used to restrict the road types or to include some special data such as lane changes and lane positions. In addition, in the collected data, the amount of drowsy driving data is low. Therefore, using a dataset comprising data with such characteristics is not recommended as the reliability of the detection technique can be affected [34].

This study analyzed the characteristics of drowsy driving and categorized them into two types: long-duration drowsy driving and short-duration drowsy driving. To effectively deal with the two types of drowsy driving, an ensemble network that consists of subnetworks based on convolution neural network (CNN), specialized for each type, is presented. By performing a time series data analysis, this study discovered and applied meaningful feature sets based on the type of drowsy driving. In addition, an imbalanced data-handling method is proposed to efficiently train the network. We collected driving data from 17 people of various ages, and the performance of the proposed network was verified with the dataset. Using the collected data, the performance of the proposed network was compared with the existing studies and it demonstrated excellent performance.

The main contributions of this study are summarized as follows:

1. A novel method to detect drowsy driving that is based on an ensemble CNN that uses vehicle internal sensor information is presented. 
2. An analysis of various angles to find the characteristics of drowsy driving, an analysis of the duration of drowsy driving, and a time series analysis are performed.

3. A performance evaluation is conducted through a driving simulation dataset consisting of general in-vehicle sensor data for a variety of road environments.

The remainder of this paper is structured as follows: Section 2 describes a quantitative analysis of the drowsy driving data ratio and the duration of the drowsy driving. Section 3 describes the preprocessing process, which includes an imbalanced data-handling method and the feature extraction method through a time series analysis for a meaningful feature set as well as the proposed CNN ensemble network. Section 4 presents the simulation and experimental environments and verifies the proposed ensemble network and analysis method through a performance evaluation that uses the collected data. Section 5 discusses the significance and limitations of this paper. The final section presents the conclusion of this study and discusses future work.

\section{Analysis on the Duration of Drowsy Driving}

We collected about $198.3 \mathrm{~h}$ of in-vehicle sensor data through driving simulations. A detailed description of the collected dataset is described in Section 4.1. This section describes the quantitative analysis and results performed on the collected dataset.

A quantitative analysis of the ratio between normal driving data and drowsy driving data was performed on the collected dataset. The data for normal driving and drowsy driving were measured based on the data label.

The ratios of normal driving and drowsy driving in relation to the entire data were determined. Figure 1 shows the results from analyzing the data ratio for each driver. According to Figure 1, the ratio differed depending on the driver. For examples, drivers \#7 and \#15 demonstrated more drowsy driving than normal; however, most drivers demonstrated more normal driving or they were rarely drowsy.

From the analysis results, it was confirmed that the collected dataset was imbalanced, which is approximately $30 \%$ of the total data for drowsy driving. The classes in the dataset were balanced with the imbalanced data-handling method that is introduced in Section 3.1.

In addition, a quantitative analysis on the duration of drowsy driving was performed. The duration was calculated from the start of drowsy driving to the end. Figure 2 is a histogram of the drowsiness duration of the entire dataset. This was treated as a single case, considering the data of the duration of being drowsy for more than $60 \mathrm{~s}$ to be sparse.

As shown in Figure 2, the two most frequent durations of drowsy driving were between 6 and $10 \mathrm{~s}$ and more than $60 \mathrm{~s}$. Inspired by this, we defined a short duration of drowsy driving (SD) and a long duration of drowsy driving (LD) when the durations of drowsy were less than $15 \mathrm{~s}$ and longer than $45 \mathrm{~s}$, respectively. We confirmed that SD occurred 1504 times and that LD occurred 880 times during data collection, and the data in both cases accounted for approximately $75.3 \%$ of the total drowsy driving.

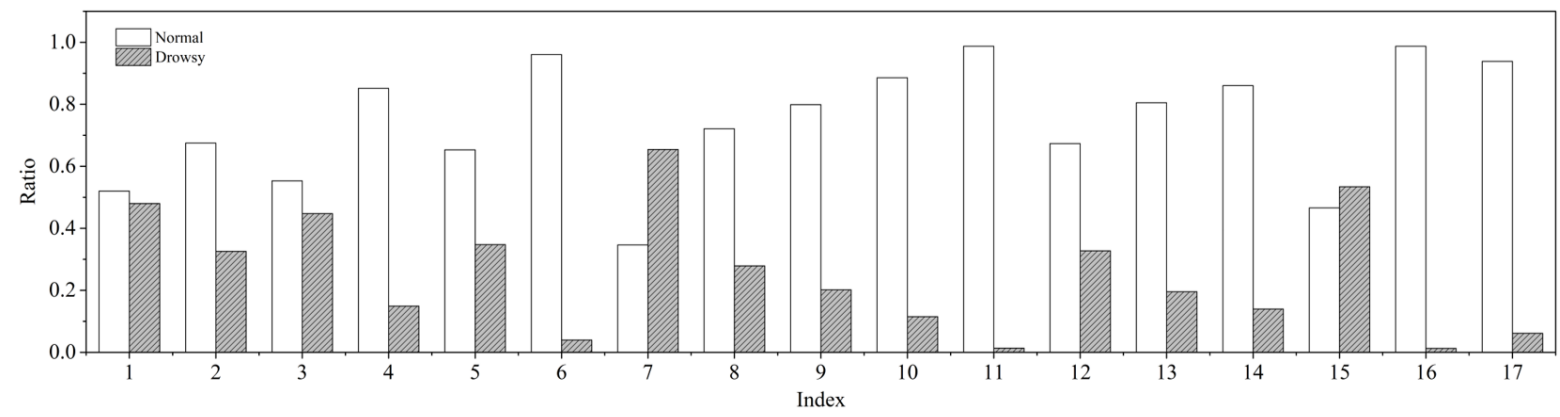

Figure 1. Ratio of normal driving and drowsy driving by driver.

Analyzing in the time domain, we observed SD for about $3.18 \mathrm{~h}$ and LD for about 14.21 h. Despite fewer occurrences of LD, it is observed for a longer time than SD because it is at least $30 \mathrm{~s}$ longer in one occurrence. 
In addition, we found drivers with a lot of SD and less LD, and vice versa. We divided drivers into two groups based on this characteristic. In the first group, the duration of drowsy driving was distributed around the SD, and there were frequent cases of drowsy driving that briefly occurred during normal driving. However, the other group showed the characteristic in which the duration of drowsy driving was distributed around a LD, and several of them fell asleep slowly and deeply after they started drowsy driving.

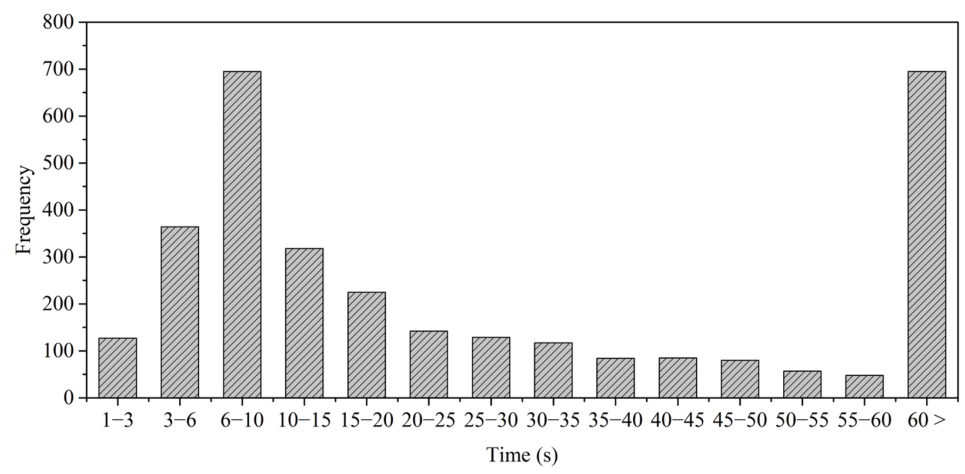

Figure 2. Histogram of the duration of drowsy driving in the dataset.

\section{Drowsy Driving Detection Method}

The proposed method for detecting drowsy driving comprises the preprocessing step, the feature extraction step, and the drowsy driving detection step. Figure 3 illustrates the overall structure of the proposed method for detecting drowsy driving. Through the preprocessing step, the data collected in Section 2 are converted into segments used for training. Subsequently, the useful features for SD and LD are searched by applying [35], which is a widely known time series analysis method. Based on the widely known time series analysis technique, a feature set specialized for SD and LD is created through useful feature search.
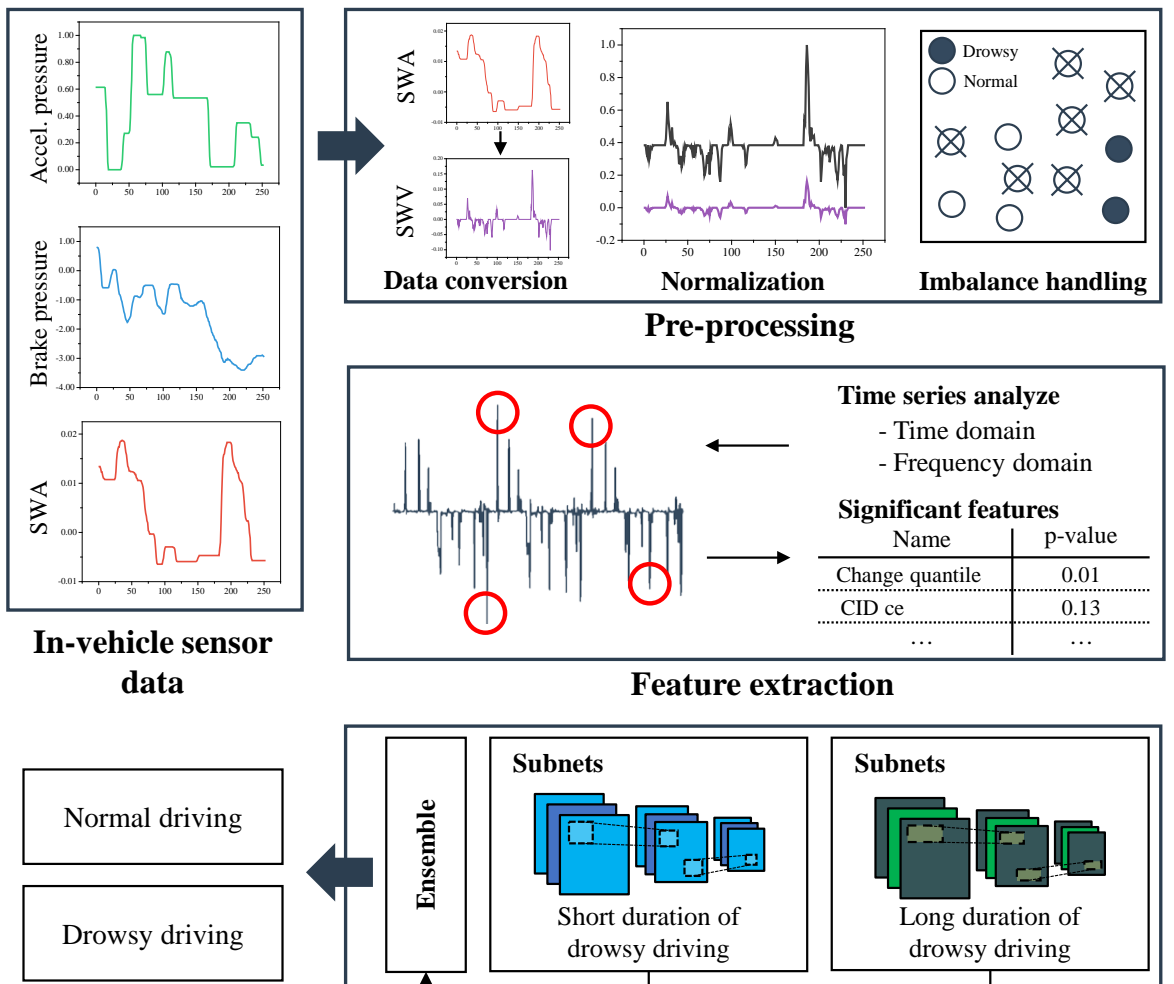

Feature extraction

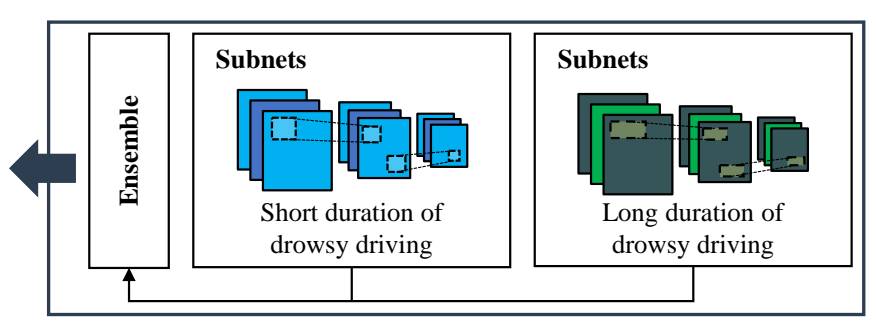

Drowsy driving detection using ensemble CNN

Figure 3. Overall process of the drowsy driving detection method. 


\subsection{Preprocessing}

The preprocessing step consists of the data conversion process, the normalization process, the imbalanced data-handling process, and the segmentation process. The steering wheel velocity (SWV) data were calculated from the steering wheel angle (SWA) data for each lap through the data conversion process. Equation (1) represents the SWV data calculation from the SWA data.

$$
S W V_{i}=\frac{S W A_{i}-S W A_{i-1}}{1 / S R}(i>0)
$$

where $S W A_{i}$ is the ith SWA value in driving. SR is the data sampling rate. In this study, since the data were acquired at $30 \mathrm{~Hz}, S R=30$ was fixed. SWV data refers to the average change rate of $S W A_{i}$ and $S W A_{i-1}$. The $S W A_{0}$ was initialized to 0.

Following this, the normalization and segmentation processes were performed. In the normalization process, the min-max normalization method was used to obtain the SWA, SWV, accelerator pedal pressure, and brake pedal pressure data.

Based on the analysis results in Section 2, an undersampling-based imbalanced datahandling method was proposed to resolve the imbalance of the dataset. The proposed method masks the drowsy driving data and the adjacent $k$ data in the driving data, as shown in Figure 4. Thus, the temporal features that appear when the state of the driver changes can be protected. Subsequently, random undersampling was performed on the unmasked data. During undersampling, $c$ consecutive data were deleted, and from this, the temporal feature of the residual data can be preserved as much as possible. Finally, the imbalance of the dataset was rechecked, and if the imbalance was still severe, undersampling was performed in the same manner.

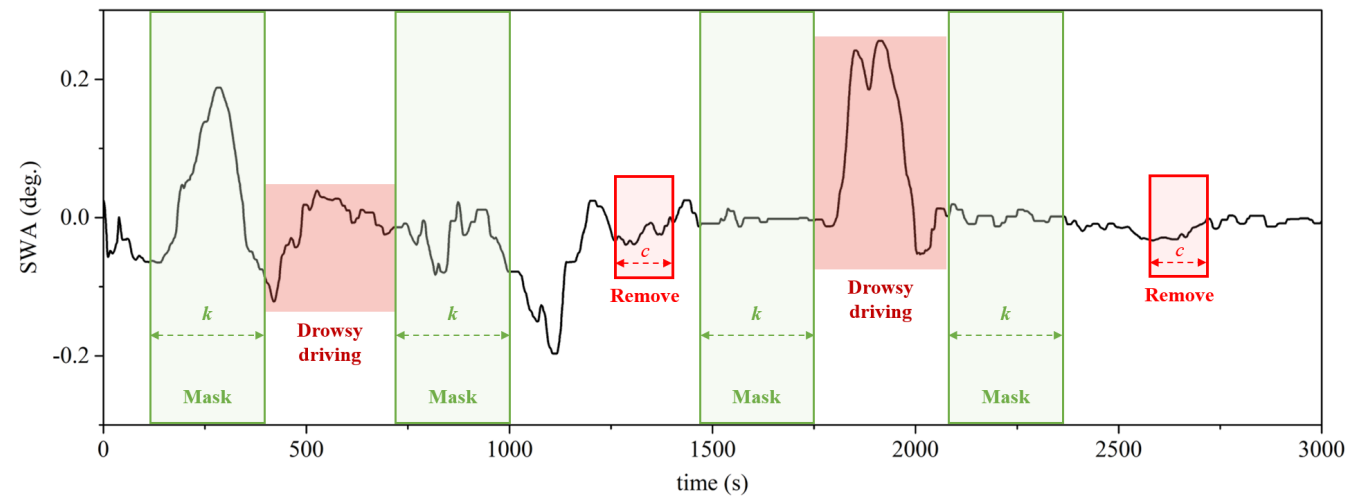

Figure 4. Mask and removal data selected by the proposed imbalanced data-handling method.

In the segmentation process, segments of a fixed size were generated through a sliding window method that is commonly used in the time series data. Parameters such as the window size $w$ and overlap size $o$ are involved in this process. We limited the window size to seconds for low computational complexity. In other words, if the $w=60$, the segment has $60 \mathrm{~s}$ of SWA, SWV, and two pedal pressure data. The overlap ratio can be calculated as $o / w$, and a high overlap ratio was designed so that many segments can have temporal features that were previously preserved.

\subsection{Feature Extraction}

We used Christ et al. [35] to find features suitable for sensor data from the vehicle through time series analysis. Christ et al. [35] presented an automated process for time series data and provided a technique for calculating $p$-values for various time series features and for selecting the significant ones. In Yadawadkar et al. [34], significant features were extracted for data such as vehicle speed and acceleration using Christ et al. [35].

We calculated $p$-values for a total of 794 time series features for the LD and SD data defined in Section 2. When the calculated $p$-value was less than 0.05 , the features were 
selected as significant features and were used to form the significant feature set for each group. Tables 1 and 2 list the significant features and $p$-values of LD and SD data.

In Tables 1 and 2, Brake refers to the brake pedal pressure and Throttle refers to the accelerator pedal pressure. SWA and SWV represent the angle and velocity of the steering wheel, respectively. The features selected as meaningful were 13 features in the LD and 57 features in the SD. More features were selected in SD, estimated to comprise a variety of meaningful features due to the relatively large amount of change in the input data.

Table 1. Significant feature list for long duration of drowsy driving (LD) data.

\begin{tabular}{llcllc}
\hline \multicolumn{1}{c}{ Type } & \multicolumn{1}{c}{ Name } & $p$-Value & Type & \multicolumn{1}{c}{ Name } & $p$-Value \\
\hline SWV & CID ce & 0.004 & Brake & Large standard deviation & 0.035 \\
SWV & Change quantiles & 0.007 & Throttle & Value count & 0.038 \\
SWV & FFT coefficient & 0.017 & Throttle & Change quantiles & 0.041 \\
Brake & AR coefficient & 0.018 & Throttle & Change quantiles & 0.042 \\
Throttle & CID ce & 0.024 & Throttle & Pct. of reoccurring data points & 0.043 \\
Throttle & Agg linear trend & 0.025 & Throttle & Pct. of reoccurring values & 0.045 \\
Throttle & FFT coefficient & 0.034 & & & \\
\hline
\end{tabular}

Table 2. Significant feature list for short duration of drowsy driving (SD) data.

\begin{tabular}{|c|c|c|c|c|c|}
\hline Type & Name & $p$-Value & Type & Name & $p$-Value \\
\hline Brake & Mean abs change & 0.003 & SWV & Mean abs change & 0.022 \\
\hline Brake & Linear trend & 0.004 & Brake & Approximate entropy & 0.024 \\
\hline Brake & Mean & 0.004 & Throttle & CID ce & 0.025 \\
\hline Brake & Ratio beyond r sigma & 0.006 & Throttle & Change quantile & 0.026 \\
\hline SWV & Kurtosis & 0.007 & Brake & Change quantile & 0.027 \\
\hline Brake & Index mass quantile & 0.008 & SWV & AR coefficient & 0.029 \\
\hline Brake & Longest strike below mean & 0.009 & Brake & C3 lag & 0.029 \\
\hline Throttle & Linear trend & 0.011 & SWA & AR coefficient & 0.031 \\
\hline Brake & Count below mean & 0.012 & SWV & Change quantiles & 0.032 \\
\hline Brake & Longest strike above mean & 0.012 & SWV & Mean abs change & 0.032 \\
\hline Brake & Number cwt peaks & 0.012 & Brake & Ratio value num. to time series & 0.032 \\
\hline SWA & CID ce & 0.013 & Brake & Absolute sum of changes & 0.033 \\
\hline Throttle & Agg linear trend & 0.013 & SWV & Skewness & 0.034 \\
\hline Brake & Pct. of reoccurring data points & 0.013 & SWV & Ratio beyond r sigma & 0.034 \\
\hline Brake & CID ce & 0.014 & Brake & Large standard deviation & 0.034 \\
\hline Brake & Partial autocorrelation & 0.014 & Brake & Sum values & 0.040 \\
\hline SWA & Change quantiles & 0.015 & SWV & FFT coefficient & 0.041 \\
\hline Brake & Autocorrelation & 0.015 & Brake & Augmented dickey fuller & 0.041 \\
\hline Throttle & FFT coefficient & 0.016 & Brake & Count above mean & 0.041 \\
\hline SWV & Standard deviation & 0.017 & Brake & Sum of reoccurring data points & 0.041 \\
\hline SWV & Variance & 0.017 & Brake & Number crossing & 0.042 \\
\hline SWA & Number crossing & 0.017 & Brake & Abs energy & 0.043 \\
\hline Brake & Pct. of reoccurring values & 0.017 & Brake & First location of maximum & 0.043 \\
\hline SWV & Linear trend & 0.018 & Brake & Standard deviation & 0.043 \\
\hline Brake & Symmetry looking & 0.018 & Brake & Variance & 0.043 \\
\hline Brake & FFT aggregated & 0.019 & Brake & Binned entropy & 0.046 \\
\hline Brake & FFT coefficient & 0.021 & Brake & Spkt welch density & 0.046 \\
\hline Brake & Value count & 0.021 & Brake & Sum of reoccurring values & 0.047 \\
\hline SWV & Absolute sum of changes & 0.022 & & & \\
\hline
\end{tabular}

We observed the features with high frequency in the selected feature set and analyzed their suitability for the drowsy driving data. First, the change quantile feature was found to be the most selected feature in LD and SD. In LD and SD, it was confirmed that the change quantile was selected as an important feature from the brake pedal pressure data, accelerator pedal pressure data, and SWV data. Change quantile was selected as an important feature in SD of the SWA data. For this reason, we speculate that the change 
quantile feature contains the most amount of information on drowsy driving. The change quantile is an average of the absolute change in data within the corridor, which sets centering the specific sensor data value. This feature is useful when the data changes severely in a small range and when outliers exist. In the case of in-vehicle sensor data, there are many small changes during normal driving. However, during drowsy driving, the driver usually turns the steering wheel rapidly or steps on the pedal suddenly, and these behaviors appear as outliers.

CID ce [36], Fast Fourier Transform (FFT) coefficient, and aggregation linear trend were commonly selected. CID ce calculates the complexity of time series data utilizing the fact that more peaks and valleys appear as the complexity and variability of the data increases. The CID ce values in LD data were found to have a decreasing trend, and on the contrary, these values showed a tendency to increase in SD data. These trends indicate the sudden steering wheel and pedal operations that are often observed when the condition of the driver changes.

The FFT coefficient is a classic feature that can express time series data and can convert it into frequency components. Naturally, frequent vehicle internal sensor data performed during normal driving appears as high-frequency data. Conversely, when the vehicle is not manipulated during drowsy driving, the data appears in a low-frequency form.

An aggregated linear trend divides segments into smaller chunks and calculates the average value within the chunk. We constructed a linear least squares regression model for representative values of the calculated chunks and then calculated the standard deviation. The value of this feature increases when it is difficult to predict, such as in cases where data change rapidly or irregularly. On the contrary, the value of the feature decreases when it is easy to predict, such as in cases where data change monotonically. In other words, this feature well indicates when it is hard to manipulate the vehicle due to drowsy driving.

\subsection{Proposed Ensemble CNN}

We designed an ensemble network that is composed of CNN-based subnetworks specialized for LD and SD. Figure 5 shows the proposed network structure. The structure and size of the proposed network can be changed by adjusting the number of subnetworks specialized for SD detection $(S)$ and the number of subnetworks specialized for LD detection $(L)$.

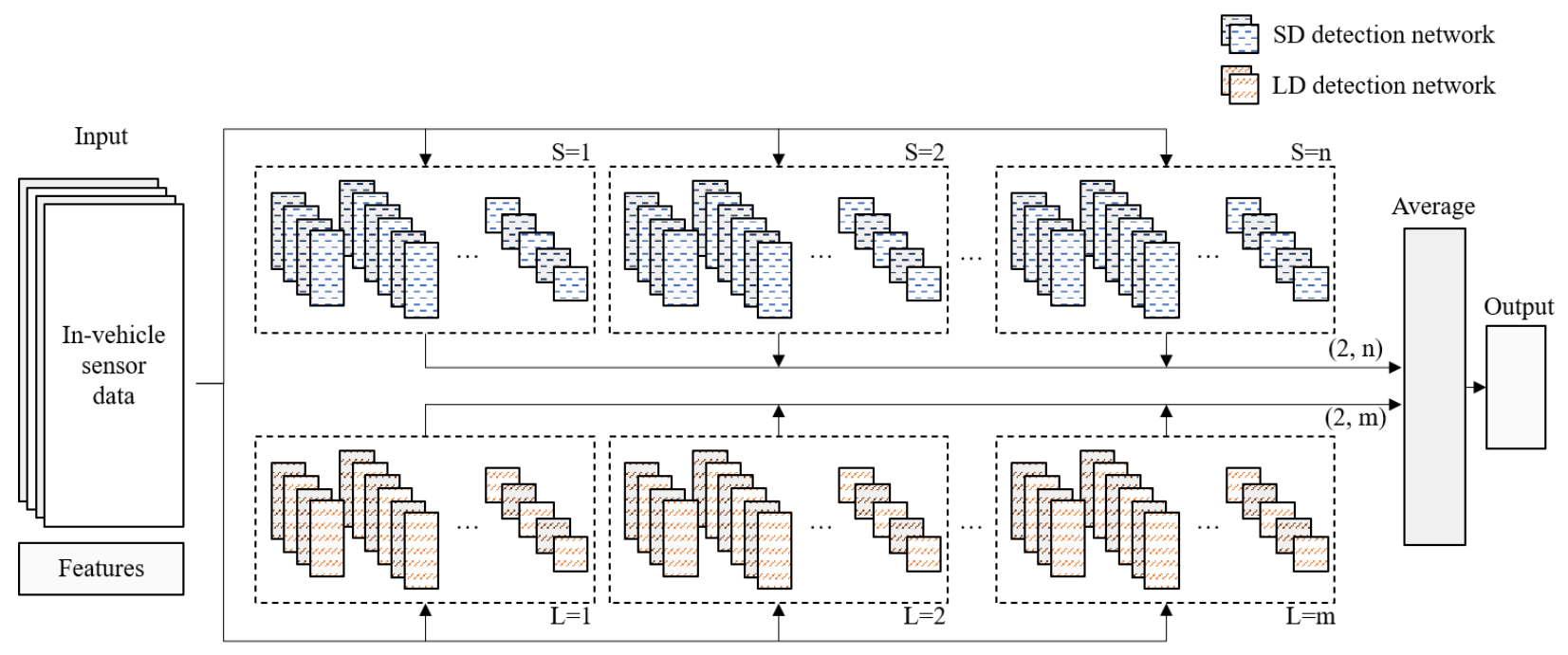

Figure 5. Proposed ensemble convolution neural network (CNN) architecture.

The proposed network utilizes the segment that is output from the preprocessing step and the features that are output from the feature extraction step as inputs. First, the proposed network transforms the segment into a four-channel 2D matrix. The segment contains $(S R \times w)$ of SWA, SWV, brake pedal pressure, and accelerator pedal pressure 
data, where $S R$ is the sampling rate and $w$ is the window size. Our proposed network transforms it into a matrix that a has $(S R \times w) \times(4)$ shape.

Through this transform, we tried to extract the convolutional features not only from adjacent data but also from data after a while. The transformed matrix and statistical features are transmitted to each subnetwork to obtain the probability of normal driving and drowsy driving, respectively. After that, the results are synthesized through the arithmetic mean to determine whether the input data is drowsy.

The CNN-based subnetwork contains three main features of VGG16 [37]. Figure 6 shows the structure of the subnetwork and the stacked convolution layer in the first block. The convolution-pooling layer structure was applied in the first, second, and third blocks, and it can be observed that the depth gradually increases. Through these structural advantages, the semantic features were effectively extracted from the vehicle sensor data. The statistical features and semantic features were fused in the last fully connected layer. The features that are listed in Table 1 were fused to the subnetwork for the LD detection, and the features listed in Table 2 were fused to the subnetwork for the SD detection. In feature fusion, a simple concatenation operation was used between the semantic and statistical features.

To design a network with good performance, it is necessary to lower the reducible error.

Reducible errors consist of bias and variance, which generally exist in a tradeoff relationship. A high bias means that all the information in the dataset were not considered. Conversely, a high variance refers to a state in which generality is lost due to excessive consideration of dataset information.

The proposed network attempts to reduce bias through a sufficiently deep subnetwork and to reduce variance through a bagging-based technique for each subnetwork. The traditional bagging technique randomly samples training data and divides it to create different subnetworks. In this paper, data from the LD group and SD group, which have different tendencies, were first divided to suppress correlations between subnetworks and to greatly reduce the variance.

Then, $m$ different networks for LD detection and $n$ different networks for SD detection were pretrained using data of LD group and SD group, respectively. Through this pretrain process, a network group that is specialized for SD and LD detection was acquired.

Subsequently, the proposed ensemble network was trained on the entire training dataset. This manner of learning may cause overfitting concerns; however, the data characteristics of LD and SD are different, and overfitting was suppressed by using enough data.

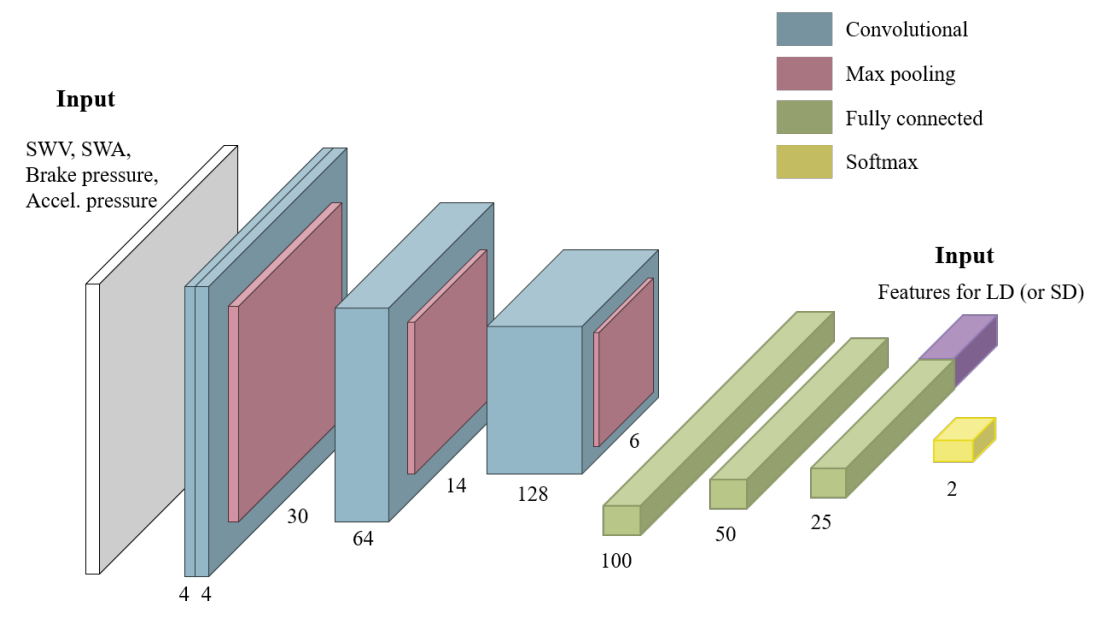

Figure 6. Proposed subnetwork architecture.

\section{Evaluation}

In this section, we describe the experimental environment and various experiment results for the performance evaluation of the proposed method. We determine the optimized value of the overlap parameter described in Section 3.1. We evaluate the impact 
of the proposed imbalanced data-handling method. We also perform an ablation study on the extracted features during the feature extraction process. Finally, we evaluate the performance according to the structure of the proposed ensemble network and compare the performance with exiting studies.

\subsection{Driving Simulation Environment}

We developed a PC-based driving simulator to acquire driving data. We installed the Logitech G920 steering wheel to drive the vehicle in the simulation. The Euro Truck Simulator 2 (ETS2) that was used in the simulator includes a wide variety of roads in Europe, which were closely simulated.

We used a vehicle developed using an extension interface called modification (MOD) for data collection. We referred to the specifications of a Hyundai Elantra model: size, acceleration, maximum speed, and rotation radius. Figure 7 shows a participant collecting data in the developed simulator.

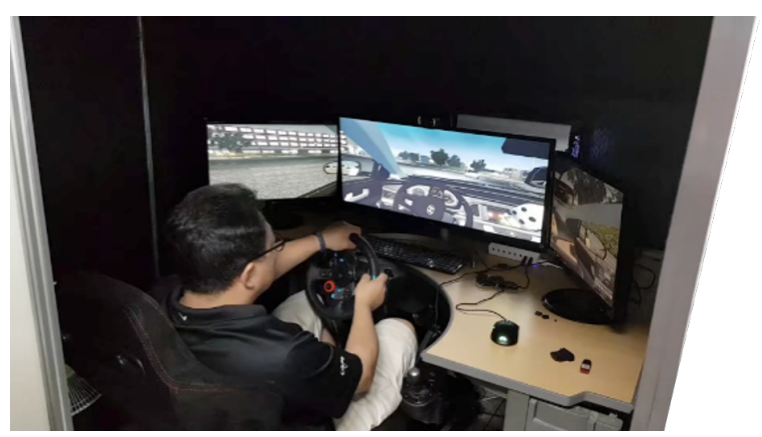

Figure 7. A participant driving in the driving simulator.

Additionally, we designed the route using the roads included in the software. The driving route for the data acquisition was set up as shown in Figure 8a. It included urban roads and highways, as shown in Figure 8 b,c. In addition, it comprised linear driving and curved driving, as shown in Figure 8d. Road structures such as toll gates, traffic lights, and interchanges, which are shown in Figure 8e, were included. Sensor data such as the SWA, accelerator pedal pressure, and brake pedal pressure during driving were acquired at a sampling rate of $30 \mathrm{~Hz}$. The driver's face was simultaneously recorded for the ground truth.

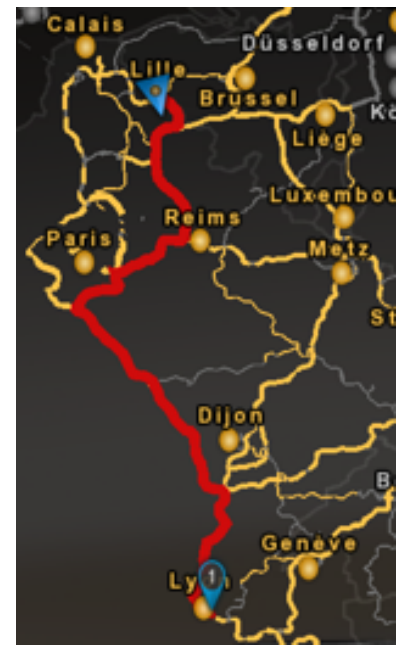

(a)

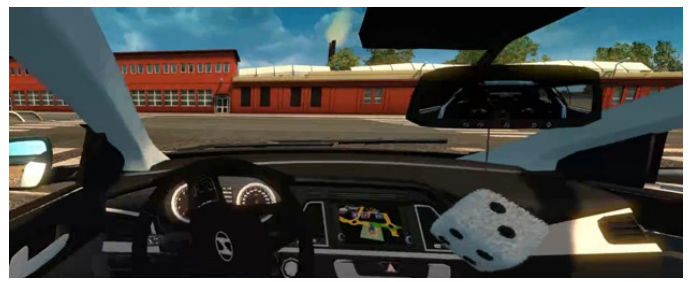

(b)

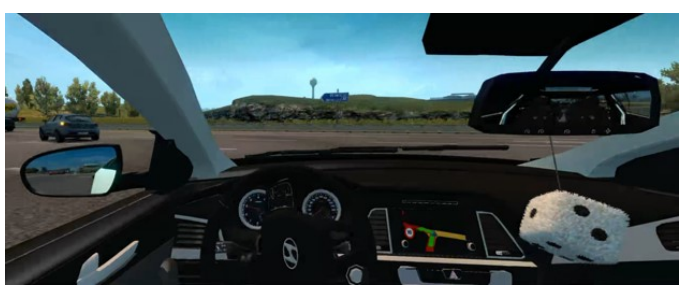

(d)

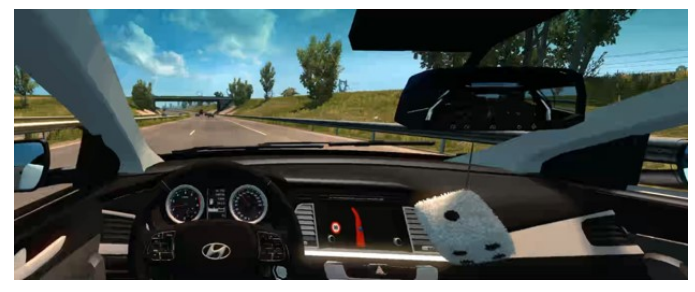

(c)

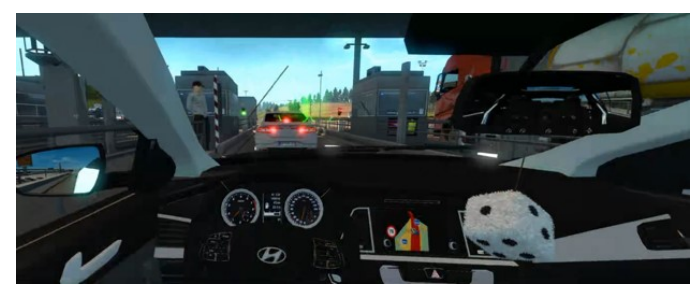

(e)

Figure 8. Driving route and road types included in simulation: (a) driving route for data acquisition, (b) urban road, (c) highway, (d) crossroad, and (e) toll gates. 
To induce drowsy driving when collecting data, we performed simulations in a dark environment where light was blocked between 2 a.m. and 4 a.m. In addition, we advised data collection participants to limit sleep-disrupting behaviors such as naps and caffeine intake before data collection. Preliminary data collection was conducted for 54 participants (21 men and 33 women), who were in their $20 \mathrm{~s}$ and $30 \mathrm{~s}$, with more than 1 year of driving experience. Participants who collected inappropriate data due to events such as deviation, speeding, and forward collision; those who were not sleepy at all; and those with mild motion sickness were excluded. As a result, 17 participants (11 males and 6 females) were selected and their data were collected. The driving route was repeatedly driven 20 times, and a total of $198.3 \mathrm{~h}$ of simulation data were collected.

Three experts labeled the drowsy state (in seconds) based on the facial movements and expressions of the driver [38,39] in the recorded video. The detailed criteria that were used for labeling are shown in Table 3.

Table 3. Criteria for determining drowsy driving according to driver behavior.

\begin{tabular}{cl}
\hline State (Value) & \multicolumn{1}{c}{ Indicators } \\
\hline Normal (0) & Eyes kept open, head in line, rapid eyeball move- \\
& ment, looking around \\
Drowsy (1) & Face stretching, yawning, sighing, intentional blink- \\
& ing of eyes, swallowing, face moving up and down \\
& (head-banging), slowing down of the eye blink, de- \\
& creased degree of an open eyelid, open eyes con- \\
& sciously, fully closed eyes, slanted head, dozing \\
\hline
\end{tabular}

\subsection{Experimental Environment}

We performed the performance evaluation on a PC with an Intel i7-7700 CPU and two Geforce GTX 2080 Ti installed. Additionally, we used the Tensorflow r1.15 and Keras packages in Ubuntu 16.04 environment to implement the proposed ensemble CNN. The application programming interface (API) of the Keras package was utilized for training, validating, and testing the models used in the experiments.

To evaluate the performance of the proposed method in detecting drowsy driving, we ran a hold-out cross validation study. The collected dataset were randomly separated into training, validation, and testing datasets with a ratio of 6:2:2. In other words, we separated the collected dataset across all participants into three subsets.

The performance of the proposed network was evaluated in terms of accuracy, precision, recall, and F1-score. The accuracy was defined as $(T P+T N) /(T P+T N+F P+$ $F N)$, and the precision was defined as $T P /(T P+F P)$. The recall was $T P /(T P+F N)$, and the F1-score was calculated as the harmonic average of the accuracy and recall.

The definitions of TP, TN, FP, and TN are as follows:

- $\quad$ True Positive (TP): Actual state is drowsy driving and inferred as drowsy driving.

- $\quad$ True Negative (TN): Actual state is normal driving and inferred as normal driving.

- $\quad$ False Positive (FP): Actual state is normal driving but inferred as drowsy driving.

- $\quad$ False Negative (FN): Actual state is drowsy driving but inferred as normal driving.

Accuracy refers to the percentage of correct detection for the drowsy driving case and normal driving case and implies the accuracy of the network production. Precision represents the ratio of drowsy driving for the cases that were detected as drowsy driving. That is, if the precision is high, the reliability of the detection result is high. Recall refers to the rate that is detected by drowsy driving among the drowsy driving cases. If the recall is high, the network can detect drowsy driving successfully. In general, recall and precision have an inverse relationship, and precision and recall can be simultaneously considered through the F1-score. The parameters used in the experiment are shown in Table 4. 
Table 4. Parameter list and value in experiments.

\begin{tabular}{clc}
\hline Parameter & Description & Value \\
\hline $\mathrm{k}$ & Mask size & 60 \\
$\mathrm{C}$ & Consecutive data length for undersampling & 5 \\
$\mathrm{~W}$ & Window size for segmentation & 60 \\
epoch & Training epoch & 500 \\
batch & Training batch size & 64 \\
\hline
\end{tabular}

\subsection{Segmentation Parameter Optimization}

The overlap parameter is a very important parameter in dealing with sequential data, and we conducted an experiment to find an appropriate value of the overlap parameter $o$. Figure 9 shows the change in accuracy according to the overlap ratio calculated by $o / w$ mentioned above using the proposed subnetwork. Until the overlap ratio reached about $84 \%$, the accuracy of the learned network tended to decrease as the overlap ratio increased. However, it was confirmed that the performance increased sharply from about $84 \%$ or more, and the best performance was shown when $o=59$. In the subsequent experiment, $o=59$ was set.

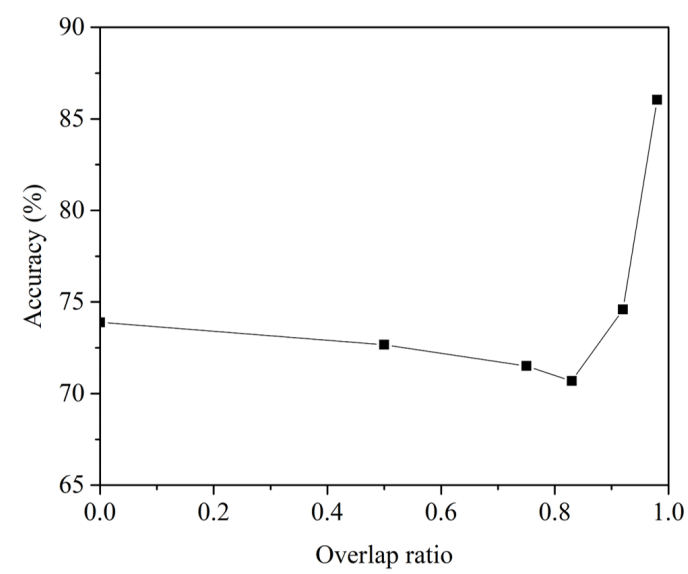

Figure 9. Accuracy of subnetwork according to overlap ratio $(w=60)$.

\subsection{Applying the Imbalanced Data-Handling Method}

First, we confirmed that, when the imbalanced data-handling method was applied, the percentage of normal driving in the entire dataset decreased from $70.46 \%$ to $53.27 \%$. It can be observed that the proposed imbalanced data-handling method effectively controls the ratio of normal driving and drowsy driving in the dataset.

To evaluate the impact of the proposed imbalanced data-handling method, we created two datasets: a balanced dataset and an imbalanced dataset. The imbalanced dataset is the original dataset collected during driving simulation. The balanced dataset is the results from applying the proposed imbalanced data-handling method on the original dataset.

We evaluated the impact of the proposed imbalanced data-handling method by comparing the performance of two networks trained with each dataset, and the results are shown in Table 5. Regarding the network architecture parameters defined in Section 3.3, $L=4$ and $S=4$ were used.

The accuracies of the network trained with the balanced dataset and of the network trained with the imbalanced dataset were $93.38 \%$ and $94.10 \%$, respectively. However, we confirmed that the recalls of the network trained with the balanced dataset and of the network trained with the imbalanced dataset were $92.79 \%$ and $88.40 \%$, respectively.

This implies that the network trained with the imbalanced dataset can determine the normal driving data well but cannot determine the drowsy driving data correctly. A high recall is critical for driver safety due to the characteristics of the detection application for 
drowsy driving. Therefore, it is necessary to train the network with the balanced dataset that applies the proposed imbalanced data-handling method.

Table 5. Normalized confusion matrix of networks trained with the balanced dataset and the imbalanced dataset.

\begin{tabular}{ccccc}
\hline & \multicolumn{4}{c}{ Predicted Class } \\
\hline \multirow{2}{*}{ Actual class } & \multicolumn{2}{c}{ Balanced dataset } & \multicolumn{2}{c}{ Imbalanced dataset } \\
\cline { 2 - 5 } & Normal & Drowsy & Normal & Drowsy \\
\hline Normal & 0.940 & 0.060 & 0.998 & 0.002 \\
\hline Drowsy & 0.072 & 0.928 & 0.116 & 0.884 \\
\hline
\end{tabular}

\subsection{Ablation Study on Extracted Feature Set}

Next, an ablation study was performed on the extracted significant feature set. We removed the features with $p$-values closer to the threshold and left $n$ features. We defined these $n$ features as top $n$ features. When measuring the performance, $L=4$ and $S=4$ were used as the network architecture parameters, and Table 6 shows the results of the experiment.

An accuracy of $87.69 \%$ was achieved when 58 features were used, which exclude the change quantile feature when considering the extracted features. When the extracted features used the top 58 features that include the change quantile, the accuracy was $91.55 \%$. From this, it can be observed that the change quantile is a feature that expresses a relatively large amount of information.

When the top 34 features were used, an accuracy of $84.24 \%$ and a F1-score of $82.40 \%$ were recorded. When the features were not used, the accuracy was $82.59 \%$ and the F1-score was $82.47 \%$. This study confirmed that the accuracy and F1-score decreased as the number of used features decreased.

Table 6. Performance comparison of the ablation study on a significant feature set.

\begin{tabular}{clcc}
\hline \# of Features & \multicolumn{1}{c}{ Description of the Feature Set } & Accuracy (\%) & F1-Score (\%) \\
\hline 70 & All features (long: 13, short: 57) & 93.38 & 93.34 \\
58 & All features without Change quantiles & 87.69 & 85.46 \\
58 & Long: Top 9 features, Short: Top 49 features & 91.55 & 90.31 \\
34 & Long: Top 6 features, Short: Top 28 features & 84.24 & 82.40 \\
0 & None & 82.59 & 82.47 \\
\hline
\end{tabular}

\subsection{Experiment Result for the Proposed Ensemble CNN}

The performance was compared according to the number of subnetworks in the proposed network. Tables 7 and 8 show the normalized confusion matrix according to the proposed network architecture. The network with $L=1$ and $S=1$ showed the worst performance, with an $86.90 \%$ accuracy and an $87.07 \%$ F1-score. In the case of $L=2$ and $S=2$, the accuracy was $90.81 \%$ and the F1-score was $90.67 \%$. When $L=4$ and $S=4$, the accuracy was $93.38 \%$ and the F1-score was $93.34 \%$. As the number of subnetworks that were included in the network increased, the accuracy and F1-score tended to increase.

This trend was maintained even as the number of subnetworks further increased. In the case of $L=6$ and $S=6$, the accuracy was $93.97 \%$, and the F1-score was $93.95 \%$. In the case of $L=8$ and $S=8$, the accuracy was $94.07 \%$, and the F1-score was $94.05 \%$. When $L=10$ and $S=10$, the best performance was achieved with an accuracy of $94.20 \%$ and a F1-score of $94.18 \%$. However, we confirmed that, after $L=6$ and $S=6$, the rate of increase in the performance decreased, and it did not increase significantly even when it became larger than $L=10$ and $S=10$. 
Table 7. Normalized confusion matrix of the proposed network with a symmetric parameter.

\begin{tabular}{ccccccc}
\hline \multicolumn{7}{c}{ Predicted Class } \\
\hline \multirow{2}{*}{ Actual class } & \multicolumn{7}{c}{$(L=1, S=1)$} & \multicolumn{2}{c}{$(L=2, S=2)$} & \multicolumn{2}{c}{$(L=4, S=4)$} \\
\cline { 2 - 8 } & Normal & Drowsy & Normal & Drowsy & Normal & Drowsy \\
\hline Normal & 0.856 & 0.144 & 0.923 & 0.077 & 0.940 & 0.060 \\
\hline Drowsy & 0.118 & 0.882 & 0.107 & 0.893 & 0.072 & 0.928 \\
\hline \multirow{2}{*}{ Actual class } & \multicolumn{2}{c}{$(L=6, S=6)$} & \multicolumn{2}{c}{$(L=8, S=8)$} & \multicolumn{2}{c}{$(L=10, S=10)$} \\
\cline { 2 - 8 } & Normal & Drowsy & Normal & Drowsy & Normal & Drowsy \\
\hline Normal & 0.946 & 0.054 & 0.945 & 0.055 & 0.945 & 0.055 \\
\hline Drowsy & 0.067 & 0.933 & 0.064 & 0.936 & 0.061 & 0.939 \\
\hline
\end{tabular}

Table 8. Normalized confusion matrix of the proposed network with an asymmetric parameter.

\begin{tabular}{ccccccc}
\hline \multicolumn{7}{c}{ Predicted Class } \\
\hline \multirow{2}{*}{ Actual class } & \multicolumn{2}{c}{$(L=1, S=2)$} & \multicolumn{2}{c}{$(L=1, S=4)$} & \multicolumn{2}{c}{$(L=1, S=8)$} \\
\cline { 2 - 7 } & Normal & Drowsy & Normal & Drowsy & Normal & Drowsy \\
\hline Normal & 0.897 & 0.103 & 0.910 & 0.090 & 0.918 & 0.082 \\
\hline Drowsy & 0.105 & 0.895 & 0.089 & 0.911 & 0.082 & 0.918 \\
\hline \multirow{2}{*}{ Actual class } & \multicolumn{2}{c}{$(L=2, S=1)$} & \multicolumn{2}{c}{$(L=4, S=1)$} & \multicolumn{2}{c}{$(L=8, S=1)$} \\
\cline { 2 - 8 } & Normal & Drowsy & Normal & Drowsy & Normal & Drowsy \\
\hline Normal & 0.884 & 0.116 & 0.916 & 0.084 & 0.924 & 0.082 \\
\hline Drowsy & 0.118 & 0.882 & 0.079 & 0.921 & 0.081 & 0.919 \\
\hline
\end{tabular}

As the number of subnetworks increases, we observed that both training time and inference time were increased. When $L=6$ and $S=6$, it took about $18.6 \mathrm{~h}$ to train the model, and when $L=10$ and $S=10$, it took about $37.8 \mathrm{~h}$. Similarly, when $L=6$ and $S=6$, the inference time excluding feature calculation is $228 \mathrm{~ms}$, and when $L=10$ and $S=10$, it took about $459 \mathrm{~ms}$.

Table 8 is a normalized confusion matrix showing the performance when the model structure parameter is asymmetric. Previously, it was demonstrated that performance increases as the number of subnetworks increases even when the number of subnetworks is asymmetric, similar to when the number of subnetworks specialized for LD detection and subnetworks specialized for SD detection increase to the same number. When $L=1$ and $S=2, S=4$, and $S=8$, accuracies of $89.59 \%, 91.04 \%$, and $91.76 \%$ were recorded, respectively. Conversely, when $S=1$ was fixed and $L=2, L=4$, and $L=8,88.28 \%$, $91.81 \%$, and $92.15 \%$ were recorded, respectively.

We confirmed that, when $L=2$ and $S=6$, the accuracy was $92.36 \%$ and the F1-score was $92.35 \%$. In the case of $L=3$ and $S=5$, the accuracy was $92.92 \%$ and the F1-score was $92.83 \%$. If the value of the parameter is reversed, the performance changes slightly. When $L=6$ and $S=2$, the accuracy was $92.69 \%$ and the F1-score was $92.71 \%$. When $L=5$ and $S=3$, the accuracy was $92.71 \%$ and the F1-score was $92.95 \%$.

Figure 10 is a graph that summarizes the changes in accuracy and F1-score according to the changes in the model structure parameters $L$ and $S$ mentioned above. In each graph, the size of the dot represents the size of the model and the label represents the parameter value. A square dot means a case where $L=S$, and a triangle point means a case where $L>S$. Finally, the circular dot represents the case where $L<S$.

When the number of subnetworks in the model structure parameter is less than 8 , the performance increases rapidly as the number of subnetworks increases. In the case of 
$L=S$, it was confirmed that the range of performance increase was greater than in the case of asymmetrical number of subnetworks.

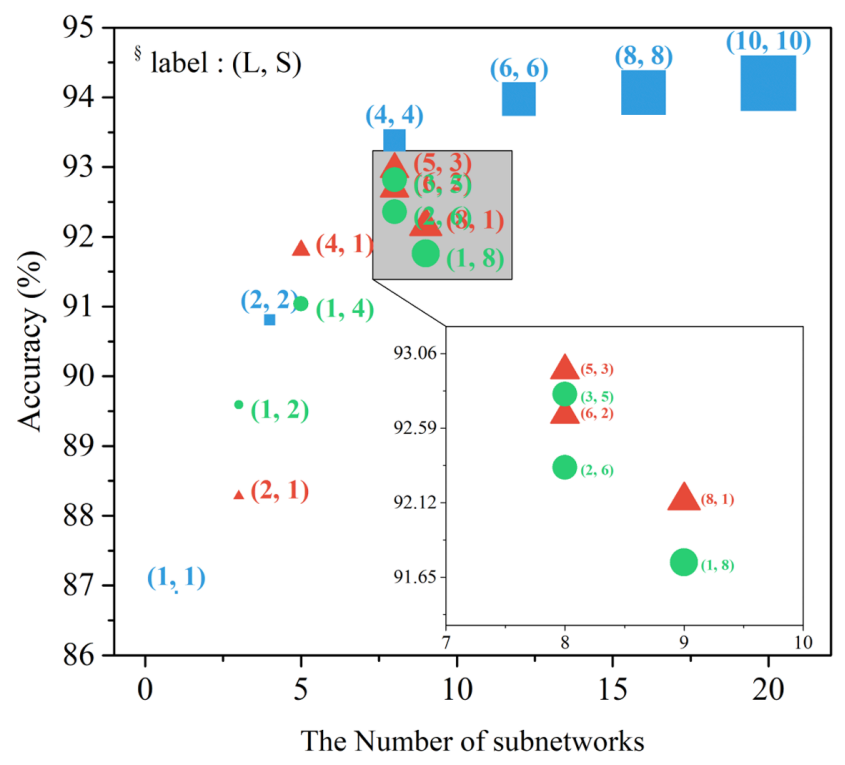

(a)

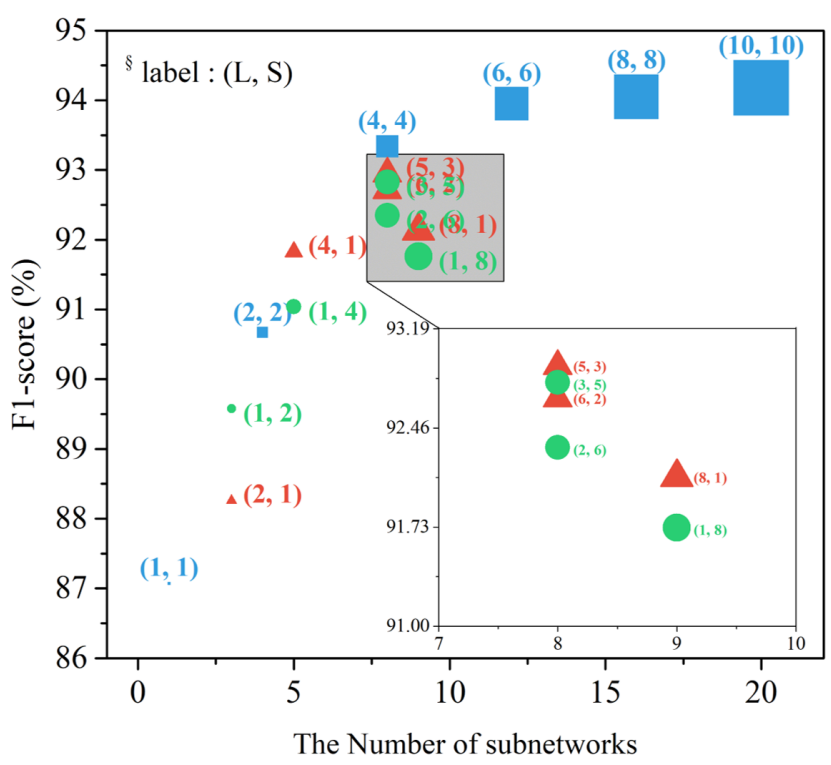

(b)

Figure 10. Performance comparison according to model structure parameter changes: (a) accuracy and (b) F1-score.

On the contrary, when the number of subnetworks is 8 or more, the performance change is not large as the number of subnetworks increases, but it can be assumed that the variance in the network is sufficiently reduced through the ensemble technique.

Even when the number of subnetworks is asymmetric, it has been shown that the performance increases as the number of subnetworks increases. It was confirmed that the case of $L>S$ has a better performance than the opposite case. According to the analysis results in Section 2, the collected dataset has more LD data than SD data, and we segmented the same window size $(w)$. Therefore, it is estimated that it is more advantageous to detect LD better than SD.

Table 9. Performance comparison with other approaches.

\begin{tabular}{|c|c|c|c|c|}
\hline Method & Accuracy (\%) & Precision (\%) & Recall (\%) & F1-Score (\%) \\
\hline Proposed & 94.20 & 93.90 & 94.47 & 94.18 \\
\hline DNN & 70.47 & 69.38 & 73.27 & 71.27 \\
\hline 1D-CNN & 72.79 & 68.02 & 61.85 & 64.79 \\
\hline LSTM & 77.13 & 77.14 & 77.10 & 77.12 \\
\hline Random forest & 72.66 & 72.77 & 8.04 & 14.48 \\
\hline
\end{tabular}

Finally, the performances of the traditional machine learning techniques, other deep learning networks, and the previous studies were compared. Table 9 shows the results of the comparison between the machine learning techniques and the other networks. We used $L=10, S=10$ as the architecture parameters of the proposed network. The deep neural network (DNN) for the performance comparison used a network in which the fully connected (FC) layer was overlapped five times. The output sizes of each layer were set as $128,256,256,64$, and 2 . We set the activation function of the last layer to a softmax function for the detection result.

One-dimensional CNN consists of two 1D convolution layers and the two FC layers. The parameters of the convolution layers used in 1D-CNN are as follows: the number of filters is 64 and 128, the kernel size is 2, and the stride is 1 . The output sizes of two FC layers are 64 and 2, and the activation function of the last layer used the softmax function. We 
designed the long short-term memory (LSTM) network to have a structure of 3 consecutive LSTM layers and 2 FC layers. Each LSTM layer had 300 units, and the output sizes of the FC layer were set to 64 and 2. In the case of the random forest model, which is a machine learning technique, 100 estimators were used.

As a result of the performance comparison, it was shown that the proposed network outperformed the other networks on all the performance measures. In particular, the LSTM network has an advantageous architecture for analyzing the time-series data such as the sensor data that is used in this study. It shows a higher performance than the $\mathrm{DNN}, \mathrm{CNN}$, and random forest models; however, it has a lower performance than the proposed network.

Table 10. Performance comparison and used variables with previous studies.

\begin{tabular}{clc}
\hline Study & \multicolumn{1}{c}{ Used Variables } & Accuracy (\%) \\
\hline $\begin{array}{c}\text { Proposed } \\
{[33]}\end{array}$ & SWA, SWV, pedal pressure & 94.20 \\
\hline$[40]$ & SWA, SWV, yawn rate, lateral acceleration & 84.95 \\
{$[31]$} & SWA, pedal pressure, lane deviation & 86.10 \\
{$[29]$} & SWA & 79.00 \\
{$[27]$} & Lateral position, vehicle speed, PERCLOS & 78.01 \\
\hline
\end{tabular}

$\S$ Using the collected dataset in our study.

We also performed comparisons with previous studies. Previous studies $[27,29,31,40]$ used traditional machine learning-based methods, whereas Arefnezhad et al. [33] proposed a CNN-LSTM network to detect drowsy driving.

As shown in Table 10, SWA data are used in many studies and pedal pressure data are also used to detect drowsy driving $[29,31,33,40]$. Although not used in this paper, in-vehicle sensor data such as lateral acceleration data [33] and vehicle speed [27] are also used. In addition, deviation of the vehicle position in a lane [27,33,40] or PERCLOS [27] are used to improve the detection performance.

McDonald et al. [31] collected approximately $108 \mathrm{~h}$ of data from 72 participants. The data from the collected dataset in McDonald et al. [31] were segmented to a $60 \mathrm{~s}$ window and sampled at a reduced frequency of $1 \mathrm{~Hz}$. Zhang et al. [27] collected about $27 \mathrm{~h}$ of data from 27 participants on a circular highway track. The collected data in Zhang et al. [27] were segmented into $600 \mathrm{~s}$ non-overlapping windows. Arefnezhad et al. [33] collected data through a driving simulation from 13 participants on a monotonous highway at a sampling rate of $100 \mathrm{~Hz}$ that was used as input to the network without any preprocessing. Krajewski et al. [40] collected data from 12 participants through simulations for about 11 $\mathrm{h}$ and segmented the collected data. Each segment had a length of $240 \mathrm{~s}$. Unlike other studies, Li et al. [29] collected data from real vehicles for about $14 \mathrm{~h}$ from 6 participants. The collected data in Krajewski et al. [40] were sampled to $60 \mathrm{~s}$ interval.

In Li et al. [29], an approximate entropy feature was created from SWA data, and in Zhang et al. [27], average and standard deviation were calculated from vehicle speed and lateral position. Similarly, we extracted the approximate entropy feature from brake pedal pressure for SD detection.

We also compared the accuracy performance with the reported results of previous studies [27,29,31,40]. Additionally, the network that was proposed in Arefnezhad et al. [33] was implemented, and the performance was evaluated through the collected dataset. According to Table 10, the proposed method showed superior performance compared to other previous studies. It can be observed that the characteristics for the duration of drowsy driving that were used in this study are very effective. For example, in [40], a variety of features were extracted from several domains and used; however, the performance was relatively low, with an accuracy of $86.10 \%$.

In Arefnezhad et al. [33], the achieved accuracy was 95.05\%, but when applied to our collected dataset, it was $84.95 \%$. We speculate that these results are due to the various 
road conditions and implementation constraints included the simulation. As mentioned above, the dataset was collected on a monotonous highway at a sampling rate of $100 \mathrm{~Hz}$. The dataset in our study was collected on more complex road environments at sampling rate of $30 \mathrm{~Hz}$. In the dataset that was collected, the lateral deviation data that was used in Arefnezhad et al. [33] did not exist; thus, only the SWA, SWV, yawn rate, and lateral acceleration data were used.

\section{Discussion}

From the collected dataset, we found that the duration of drowsy driving is distributed around short and long periods of time. Inspired by this, we designed an ensemble CNN composed of subnetworks specialized for detecting short and long durations of drowsy driving. We confirmed that it is effective in detecting drowsy driving.

Previous studies have determined and utilized the unique features of vehicle control patterns to detect drowsy driving $[29,31,33]$. In this study, the significant features were obtained through time series analysis. Based on a comparative stukdy, we confirmed that it is effective to divide the drowsy driving into two classes according to duration.

We showed that the proposed ensemble CNN works well in the simulation environment with various road types, but significant research is required to apply the same to the real world. For example, an in-vehicle sensor from an actual vehicle is less accurate than a simulator. In other words, since sensor data in a vehicle is noisier than a simulator, further studies with robustness are needed.

The drowsy driving detection method should have a short inference time because the detection method must observe the changing drowsiness state of the driver in real time. In this paper, a large number of significant features and a large neural network model are used; thus, high computing power would be required to obtain a short inference time. However, it is difficult to satisfy this in an actual vehicle environment. Considering these points, we plan to study drowsy driving detection methods that can work in real vehicles as future work.

\section{Conclusions}

This study proposes a method to detect drowsy driving using vehicle sensor data that comprise the steering wheel angle, accelerator pedal pressure, and brake pedal pressure data. Drowsy driving was classified into two types, long-duration drowsy driving and short-duration drowsy driving, according to the duration of drowsiness, and an ensemble CNN composed of subnetworks specialized for each drowsy driving detection was implemented. Through a time series analysis, significant features such as change quantile, CID ce, FFT coefficient, and aggregation linear trend were extracted to describe long-duration drowsy driving and short-duration drowsy driving effectively, along with an imbalanced data-handling method. To verify the proposed model, a dataset comprising $198.3 \mathrm{~h}$ of in-vehicle sensor data was acquired through a driving simulation involving 17 participants.

The proposed ensemble CNN achieved an accuracy of up to $94.20 \%$ and a F1-score of $94.18 \%$. By performing a comparative study, it was demonstrated that the ensemble CNN has excellent performance. In addition, this study confirmed that the extracted features and imbalanced data-handling methods are effective for the proposed network in detecting drowsy driving.

Author Contributions: Conceptualization, methodology and experimental design, Y.J.; project administration and super vision, Y.B.; writing and editing, Y.J.; review: B.K. and Y.B. All authors have read and agreed to the published version of the manuscript.

Funding: This research received no external funding.

Institutional Review Board Statement: The study was conducted according to the guidelines of the Declaration of Helsinki and approved by the Institutional Review Board of Pusan National University (identification code: 2018_120_HR). 
Informed Consent Statement: Informed consent was obtained from all subjects involved in the study.

Acknowledgments: This work was supported by the Institute of Information and Communications Technology Planning and Evaluation (IITP) grant funded by the Korea government (MSIT) (No. 2020-0-01450, Artificial Intelligence Convergence Research Center (Pusan National University)).

Conflicts of Interest: The authors declare no conflict of interest.

\section{References}

1. Li, D.; Gao, H. A hardware platform framework for an intelligent vehicle based on a driving brain. Engineering 2018, 4, 464-470. [CrossRef]

2. Gao, H.; Zhu, J.; Li, X.; Kang, Y.; Li, J.; Su, H. Automatic Parking Control of Unmanned Vehicle Based on Switching Control Algorithm and Backstepping. IEEE ASME Trans. Mech. 2020, 1-10. [CrossRef]

3. Gao, H.; Zhu, J.; Zhang, T.; Xie, G.; Kan, Z.; Hao, Z.; Liu, K. Situational assessment for intelligent vehicles based on Stochastic model and Gaussian distributions in typical traffic scenarios. IEEE Trans. Syst. Man Cybern. Syst. 2020, 1-11. [CrossRef]

4. Gao, H.; Su, H.; Cai, Y.; Wu, R.; Hao, Z.; Xu, Y.; Wu, W.; Wang, J.; Li, Z.; Kan, Z. Trajectory prediction of cyclist based on dynamic bayesian network and long short-term memory model at unsignalized intersections. Sci. China Inform. Sci. 2020, 1-12. [CrossRef]

5. Xie, G.; Gao, H.; Qian, L.; Huang, B.; Li, K.; Wang, J. Vehicle trajectory prediction by integrating physics-and maneuver-based approaches using interactive multiple models. IEEE Trans. Ind. Electron. 2017, 65, 5999-6008. [CrossRef]

6. Gao, H.; Cheng, B.; Wang, J.; Li, K.; Zhao, J.; Li, D. Object classification using CNN-based fusion of vision and LIDAR in autonomous vehicle environment. IEEE Trans. Ind. Inform. 2018, 14, 4224-4231. [CrossRef]

7. Gao, H.; Guo, F.; Zhu, J.; Kan, Z.; Zhang, X. Human motion segmentation based on structure constraint matrix factorization. Inform. Sci. 2022, 65, 119103.

8. Sahayadhas, A.; Sundaraj, K.; Murugappan, M. Detecting driver drowsiness based on sensors: A review. Sensors 2012, 12, 16937-16953. [CrossRef]

9. Available online: https://www.nhtsa.gov/risky-driving/drowsy-driving (accessed on 22 January 2021).

10. Kim, K.T.; Jung, K.Y. Current status and proposal of drowsy driving. J. Sleep Med. 2016, 13, 35-39. [CrossRef]

11. Kumar, A.; Patra, R. Driver drowsiness monitoring system using visual behaviour and machine learning. In Proceedings of the 2018 IEEE Symposium on Computer Applications \& Industrial Electronics (ISCAIE), Penang, Malaysia, 28-29 April 2018; pp. 339-344.

12. Huynh, X.P.; Park, S.M.; Kim, Y.G. Detection of driver drowsiness using 3D deep neural network and semi-supervised gradient boosting machine. In Proceedings of the Asian Conference on Computer Vision, Taipei, Taiwan, 20-24 November 2016; Springer: Berlin/Heidelberg, Germany, 2016; pp. 134-145.

13. Hossain, M.Y.; George, F.P. IOT Based Real-Time Drowsy Driving Detection System for the Prevention of Road Accidents. In Proceedings of the 2018 International Conference on Intelligent Informatics and Biomedical Sciences (ICIIBMS), Bangkok, Thailand, 21-24 October 2018; Volume 3, pp. 190-195.

14. Dwivedi, K.; Biswaranjan, K.; Sethi, A. Drowsy driver detection using representation learning. In Proceedings of the 2014 IEEE International Advance Computing Conference (IACC), Gurgaon, India, 21-22 February 2014; pp. 995-999.

15. Galarza, E.E.; Egas, F.D.; Silva, F.M.; Velasco, P.M.; Galarza, E.D. Real time driver drowsiness detection based on driver's face image behavior using a system of human computer interaction implemented in a smartphone. In Proceedings of the International Conference on Information Theoretic Security, Libertad City, Ecuador, 10-12 January 2018; Springer: Berlin/Heidelberg, Germany, 2018; pp. 563-572.

16. Dornaika, F.; Reta, J.; Arganda-Carreras, I.; Moujahid, A. Driver Drowsiness Detection in Facial Images. In Proceedings of the 2018 Eighth International Conference on Image Processing Theory, Tools and Applications (IPTA), Xi'an, China, 7-10 November 2018; IEEE: Piscataway, NJ, USA, 2018; pp. 1-6.

17. Schmidt, J.; Braunagel, C.; Stolzmann, W.; Karrer-Gauß, K. Driver drowsiness and behavior detection in prolonged conditionally automated drives. In Proceedings of the 2016 IEEE Intelligent Vehicles Symposium (IV), Gothenburg, Sweden, 19-22 June 2016; pp. 400-405.

18. Chui, K.T.; Tsang, K.F.; Chi, H.R.; Ling, B.W.K.; Wu, C.K. An accurate ECG-based transportation safety drowsiness detection scheme. IEEE Trans. Ind. Inform. 2016, 12, 1438-1452. [CrossRef]

19. Lee, H.; Lee, J.; Shin, M. Using wearable ECG/PPG sensors for driver drowsiness detection based on distinguishable pattern of recurrence plots. Electronics 2019, 8, 192. [CrossRef]

20. Arefnezhad, S.; Eichberger, A.; Frühwirth, M.; Kaufmann, C.; Moser, M. Driver Drowsiness Classification Using Data Fusion of Vehicle-based Measures and ECG Signals. In Proceedings of the 2020 IEEE International Conference on Systems, Man, and Cybernetics (SMC), Toronto, ON, Canada, 11-14 October 2020; pp. 451-456.

21. Mahmoodi, M.; Nahvi, A. Driver drowsiness detection based on classification of surface electromyography features in a driving simulator. Proc. Inst. Mech. Eng. Part H J. Eng. Med. 2019, 233, 395-406. [CrossRef] [PubMed]

22. Mahmoodi, M.; Nahvi, A. Investigation of Sleep Deprivation Effect on Driver's Electromyography Signal Features in a Driving Simulator. J. Sleep Sci. 2018, 3, 53-62. 
23. Tan, Z.X.; Foong, R.; Ang, K.K. Determining mechanical and electromyographical reaction time in a BCI driving fatigue experiment. In Proceedings of the 2015 10th International Conference on Information, Communications and Signal Processing (ICICS), Singapore, 2-4 December 2015; pp. 1-5.

24. Bittner, R.; Hána, K.; Poušek, L.; Smrka, P.; Schreib, P.; Vysokỳ, P. Detecting of fatigue states of a car driver. In Proceedings of the International Symposium on Medical Data Analysis, Frankfurt am Main, Germany, 29-30 September 2000; Springer: Berlin/Heidelberg, Germany, 2000; pp. 260-273.

25. Sinha, O.; Singh, S.; Mitra, A.; Ghosh, S.; Raha, S. Development of a drowsy driver detection system based on EEG and IR-based eye blink detection analysis. In Advances in Communication, Devices and Networking; Springer: Berlin/Heidelberg, Germany, 2018; pp. 313-319.

26. Wei, C.S.; Wang, Y.T.; Lin, C.T.; Jung, T.P. Toward drowsiness detection using non-hair-bearing EEG-based brain-computer interfaces. IEEE Trans. Neural Syst. Rehabil. Eng. 2018, 26, 400-406. [CrossRef]

27. Zhang, X.; Wang, X.; Yang, X.; Xu, C.; Zhu, X.; Wei, J. Driver drowsiness detection using mixed-effect ordered logit model considering time cumulative effect. Anal. Methods Accid. Res. 2020, 26, 100114. [CrossRef]

28. Gastaldi, M.; Rossi, R.; Gecchele, G. Effects of driver task-related fatigue on driving performance. Proc. Soc. Behav. Sci. 2014, 111, 955-964. [CrossRef]

29. Li, Z.; Li, S.E.; Li, R.; Cheng, B.; Shi, J. Online detection of driver fatigue using steering wheel angles for real driving conditions. Sensors 2017, 17, 495. [CrossRef]

30. Friedrichs, F.; Yang, B. Drowsiness monitoring by steering and lane data based features under real driving conditions. In Proceedings of the 2010 18th European Signal Processing Conference, Aalborg, Denmark, 23-27 August 2010; pp. $209-213$.

31. McDonald, A.D.; Lee, J.D.; Schwarz, C.; Brown, T.L. Steering in a random forest: Ensemble learning for detecting drowsinessrelated lane departures. Hum. Factors 2014, 56, 986-998. [CrossRef]

32. Chai, M. Drowsiness monitoring based on steering wheel status. Transp. Res. Part D Transp. Environ. 2019, 66, 95-103. [CrossRef]

33. Arefnezhad, S.; Samiee, S.; Eichberger, A.; Frühwirth, M.; Kaufmann, C.; Klotz, E. Applying deep neural networks for multi-level classification of driver drowsiness using Vehicle-based measures. Expert Syst. Appl. 2020, 162, 113778. [CrossRef]

34. Yadawadkar, S.; Mayer, B.; Lokegaonkar, S.; Islam, M.R.; Ramakrishnan, N.; Song, M.; Mollenhauer, M. Identifying Distracted and Drowsy Drivers Using Naturalistic Driving Data. In Proceedings of the 2018 IEEE International Conference on Big Data (Big Data), Seattle, WA, USA, 10-13 December 2018; IEEE: Piscataway, NJ, USA, 2018; pp. 2019-2026.

35. Christ, M.; Braun, N.; Neuffer, J.; Kempa-Liehr, A.W. Time series feature extraction on basis of scalable hypothesis tests (tsfresh-A python package). Neurocomputing 2018, 307, 72-77. [CrossRef]

36. Batista, G.E.; Keogh, E.J.; Tataw, O.M.; De Souza, V.M. CID: An efficient complexity-invariant distance for time series. Data Min. Knowl. Discov. 2014, 28, 634-669. [CrossRef]

37. Simonyan, K.; Zisserman, A. Very deep convolutional networks for large-scale image recognition. arXiv 2014, arXiv:1409.1556.

38. Gwak, J.; Shino, M.; Hirao, A. Early detection of driver drowsiness utilizing machine learning based on physiological signals, behavioral measures, and driving performance. In Proceedings of the 2018 21st International Conference on Intelligent Transportation Systems (ITSC), Maui, HI, USA, 4-7 November 2018; pp. 1794-1800.

39. Wierwille, W.W.; Ellsworth, L.A. Evaluation of driver drowsiness by trained raters. Accid. Anal. Prevent. 1994, 26, 571-581. [CrossRef]

40. Krajewski, J.; Sommer, D.; Trutschel, U.; Edwards, D.; Golz, M. Steering wheel behavior based estimation of fatigue. In Proceedings of the Fifth International Driving Symposium on Human Factors in Driver Assessment, Training and Vehicle Design, Big Sky, MT, USA, 22-25 June 2009; pp. 118-124. 